\title{
Performance comparison of microwave cavities used for extraction operations
}

\section{GIACINTO D'AMORE ${ }^{1 *}$ (1), GIUSEPPE D'AURIA ${ }^{2}$, ALDO ROMANO $^{2}$ and FRANCESCO MARRA ${ }^{1}$}

\author{
${ }^{1}$ Dipartimento di Ingegneria Industriale, Università degli studi di Salerno, Fisciano, Salerno, Italy \\ ${ }^{2}$ Dipartimento di Farmacia, Università degli studi di Salerno, Fisciano, Salerno, Italy
}

\section{CONFERENCE FULL PAPER}

Received: August 11, 2020 • Accepted: October 6, 2020

Published online: November 18, 2020

(C) 2020 The Author(s)

\begin{abstract}
In the agri-food industry world, billions of tons of waste are produced every year. This represents both a direct loss (due to the failure to exploit their potential value, and their nutritional and energy content) and indirect loss, due to their necessary treatment and/or disposal. Some substances contained in the wastes, of potential high value, can be recovered by means of extraction. Conventional extraction processes involve the use of solvents, which end up requiring an additional process of separation from the solute identified as the desired product. In recent years, extraction techniques have been proposed without the use of solvents. This work compares the performance of two microwave cavities used for extraction operations, both working at $2.45 \mathrm{GHz}$.

A calorimetric analysis performed by following the heating rate and temperature evolution in rack of 25 beakers filled with $25 \mathrm{~mL}$ of water, coupled with the solution of the heat transfer balance in the system, allowed to build the spatial distribution of the electromagnetic power dissipated as heat in each of the beakers. Fluid-dynamics aspects related to the recovery of the vapour phase produced during the extraction were also analyzed, with particular emphasis on the mean residence time of the vapour fraction in the extraction chamber as a function of its configuration.
\end{abstract}

\section{KEYWORDS}

microwaves, extraction, process intensification

\footnotetext{
"Corresponding author. E-mail: g.damore10@studenti.unisa.it
} 


\section{INTRODUCTION}

According to the latest approach of chemical industrial processes, there is a claim that some basic principles have to be accomplished to respect a more general trend of clean, sustainable development (Chemat et al., 2012). Among them are energy saving, renewable sources, reduced unit operations, robust processes, that can be altogether synthetized as process intensification. Actually, extraction is one of the most difficult operations to be performed according to the recommendations above, since the tremendous impact it typically has on the environment. As a matter of fact, it requires large consumption of either energy, water in conventional plants and raw materials due to is relatively low yield.

Nevertheless, the industrial interest for extraction is growing, as new fields, such as nutraceutics and cosmeceutics areas, are now open to the bioactive compounds obtained. It has to be noted that this market. potentially enormous, requires purity grades far higher than what the chemical industry is used to accept.

To this purpose, new methods for the extraction of bioactive compounds from vegetable matrices have been proposed in the last years, all of them trying to get rid of all the drawbacks presented by either expensive, low efficiency or toxic solvents. Use of microwaves appears to overcome all the above difficulties, as the effect of interactions of electromagnetic fields with vegetable matrices is to generate an endogenous water vapor that, in turn, forms a heteroazeotrope (Berk, 2013) with essential oils and other bioactive molecules, allowing the extraction without solvents. The method seems to offer a great potential, although it requires accuracy and innovation in design and implementation of the necessary equipment.

There is evidence that the use of microwaves reduces process times, improving yields or even making possible extraction processes otherwise difficult to achieve (Robinson et al., 2010). However, the variety of possible systems (Single mode, Multimode, Traveling Wave Tubes, Cavity, Open Applicator, et caetera), makes the choice more difficult due to the presence of drawbacks in each of them. The extraction by microwaves has two crucial points: i) the field distribution, that has to be as much homogeneous as possible not to cause hot or cold spots in the sample undergoing irradiation; ii) the microwaves power control, as it drives the whole process; iii) the design of the extraction chamber, with special reference to the internal fluid dynamics of all the extracted compounds. Papers aiming at describing the field in cavities by fairly complex mathematical evaluations (Curet et al., 2008; Mendez-Sanchez et al., 2003), sometimes are not in perfect agreement with the experimental results or refer to model systems far from the real apparatuses.

This work proposes a simple but effective calorimetric method that allows to describe how the specific power - generated by the interaction of the electromagnetic field with the loaded matrix - is distributed in a given load placed in a microwave cavity. The method also highlights differences between the two cavities used in the work in terms of microwave field distribution.

\section{MATERIALS AND METHODS}

\section{The microwave systems}

In this work, the extraction by microwave has been selected considering that water present in the vegetable matrices of interest may allow to operate in a solvent-free mode. For the purposes of 
optimizing the plant for the extraction process, two microwave cavities were used and compared, with different modes of operation. The first one was a commercial type, with the magnetron working with duty-cycles defined by an operating phase at maximum power followed by one at magnetron off. The second cavity operated in a modified mode, with the magnetron constantly working during the whole process time. The following points were analyzed: i) the behavior of electromagnetic waves within the two cavities; ii) the advantage, if any, deriving from a specially developed microwaves generator with a linearly variable control of the delivered power.

The first microwave cavity used (Fig. 1), indicated by W, was a commercial-type one, $900 \mathrm{~W}$ (size $\mathrm{L}=31.7 \mathrm{~cm} ; \mathrm{H}=21 \mathrm{~cm} ; \mathrm{W}=31.5 \mathrm{~cm}$ ), equipped with a reflecting wave stirrer (rotating paddle) that homogenizes the electromagnetic field as much as possible. The second one (Fig. 2), called L (size $\mathrm{L}=25.8 \mathrm{~cm} ; \mathrm{H}=19 \mathrm{~cm} ; \mathrm{W}=30 \mathrm{~cm}$ ), was modified by replacing the original magnetron $(800 \mathrm{~W})$ with a $900 \mathrm{~W}$ one, whereas the plate rotation mechanism was removed to allow housing in safety of the extraction vessels. Moreover, a specially designed, linearly variable power supplier was added.

\section{The grid}

A simple but effective calorimetric method to study the differences between the electromagnetic fields of the two systems above is proposed. It allows to describe how the specific power (i.e. the power per unit volume) - dissipated by the interaction of the electromagnetic field with the loaded matrix - is distributed in a given load placed in the microwave cavity. To this purpose, 25 intersection points were defined on a 5 columns $\times 5$ rows grid, $25.5 \times$ $21.5 \mathrm{~cm}$. On each point a beaker was placed, filled with $25 \mathrm{~mL}$ of water. The tests were carried out by simultaneously monitoring all beakers through the use of optical fibers for

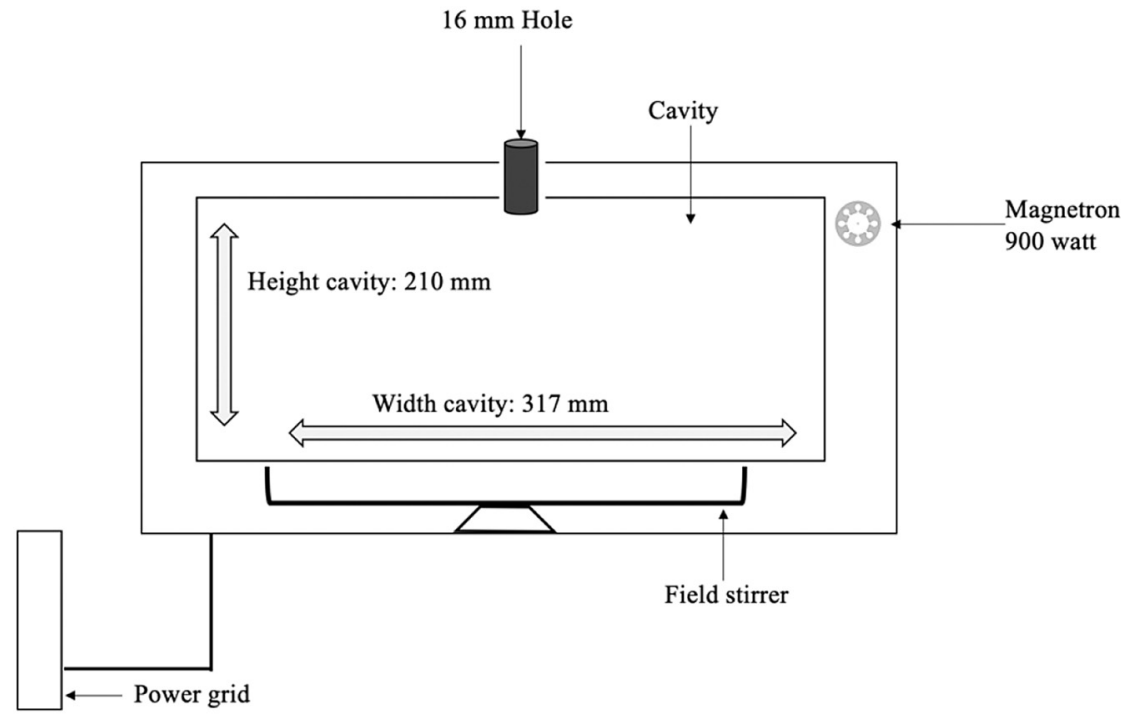

Fig. 1. Plant sketch. Cavity W 


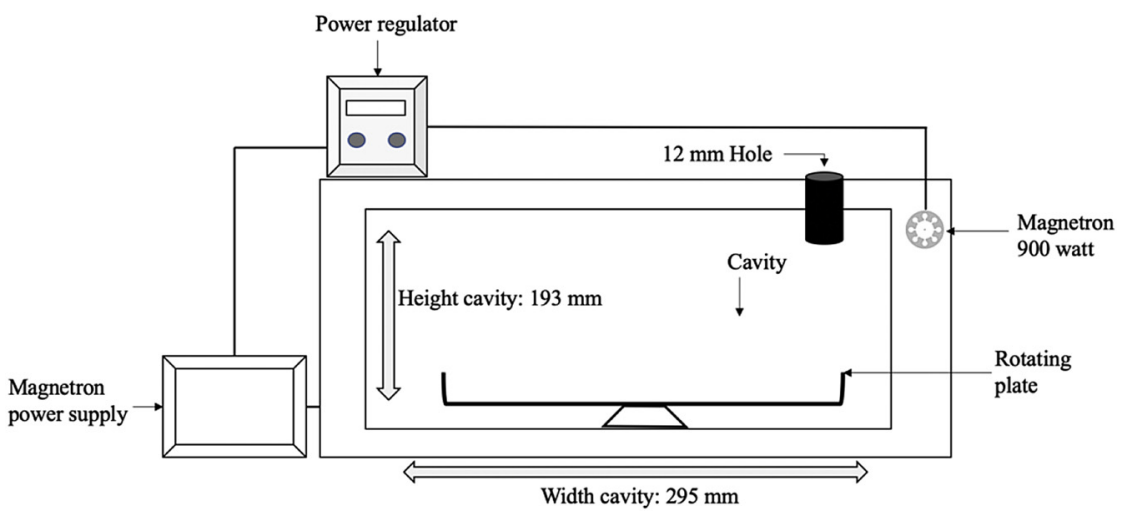

Fig. 2. Plant sketch. Cavity L

temperature measurements. The calorimetric tests were carried out on both the commercial $\mathrm{W}$ and the modified $\mathrm{L}$ cavities. The temperature measurements were carried out with a temperature sensing instrument of the company FISO, composed by a signal conditioner, a data processing program and optical fiber probes with a detectable temperature range from -40 to $300{ }^{\circ} \mathrm{C}$, a length of $2 \mathrm{~m}$, a diameter of $1 \mathrm{~mm}$ and a measurement error of $\pm 1{ }^{\circ} \mathrm{C}$.

\section{The heat balance}

Under the hypothesis that both conductive and convective contributions to the heat transfer are negligible, the heat balance allows the calculation of specific power generated by the microwaves in the water samples, $Q_{m w}$ :

$$
\rho C p \frac{\partial T}{\partial t}=Q_{m w}
$$

where $\rho$ is mass density, $C p$ is heat capacity, $T$ is temperature, $t$ is time.

Using Eq. (1), the specific power generated by the microwaves in the water samples was estimated and analyzed as function of time and space.

\section{RESULTS}

Results are shown in terms of temperature and specific power evolution and distribution in the two microwave cavities used.

The temperature-time evolution in the five beakers placed on the central column of the samples' grid in cavity $\mathrm{W}$ and cavity L, both working with a power of $350 \mathrm{~W}$, are shown in Fig. $3 \mathrm{a}$ and $\mathrm{b}$ respectively.

In Fig. $4 \mathrm{a}$ and b, the temperature distribution in cavity $\mathrm{W}$ and cavity $\mathrm{L}$ after 120 seconds of exposition to $350 \mathrm{~W}$ microwave power are shown.

The specific power distribution (computed as from Eq. (1)) in cavity W and cavity L during the exposition to an average microwave power of $350 \mathrm{~W}$ are shown in Fig. $5 \mathrm{a}$ and $\mathrm{b}$ 


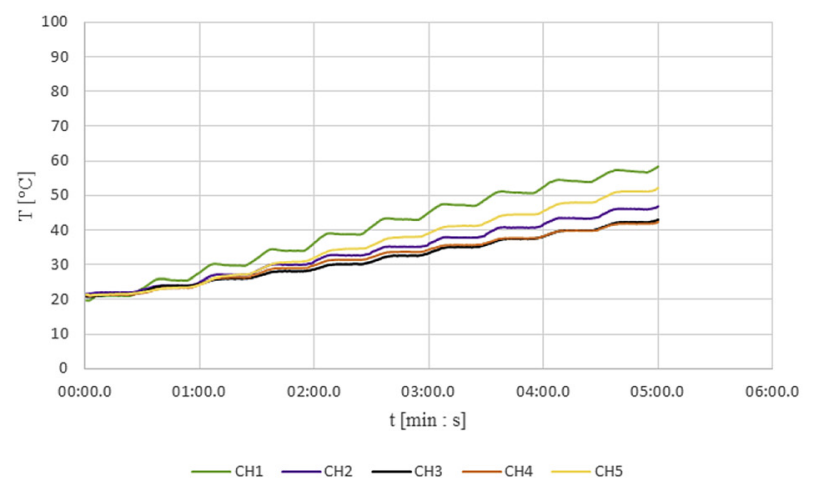

a)

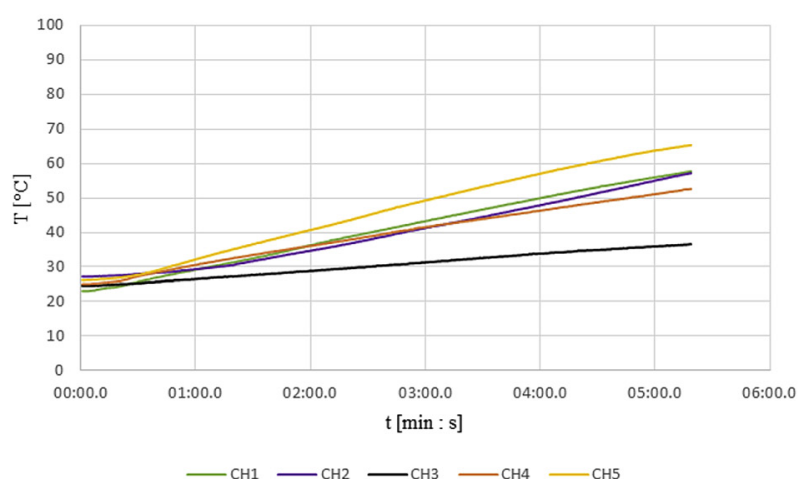

b)

Fig. 3. Temperature-time evolution in the five beakers placed on the central column of the samples' grid in cavity W (a) and cavity L (b). Channel 1 : back; Channel 5: front 


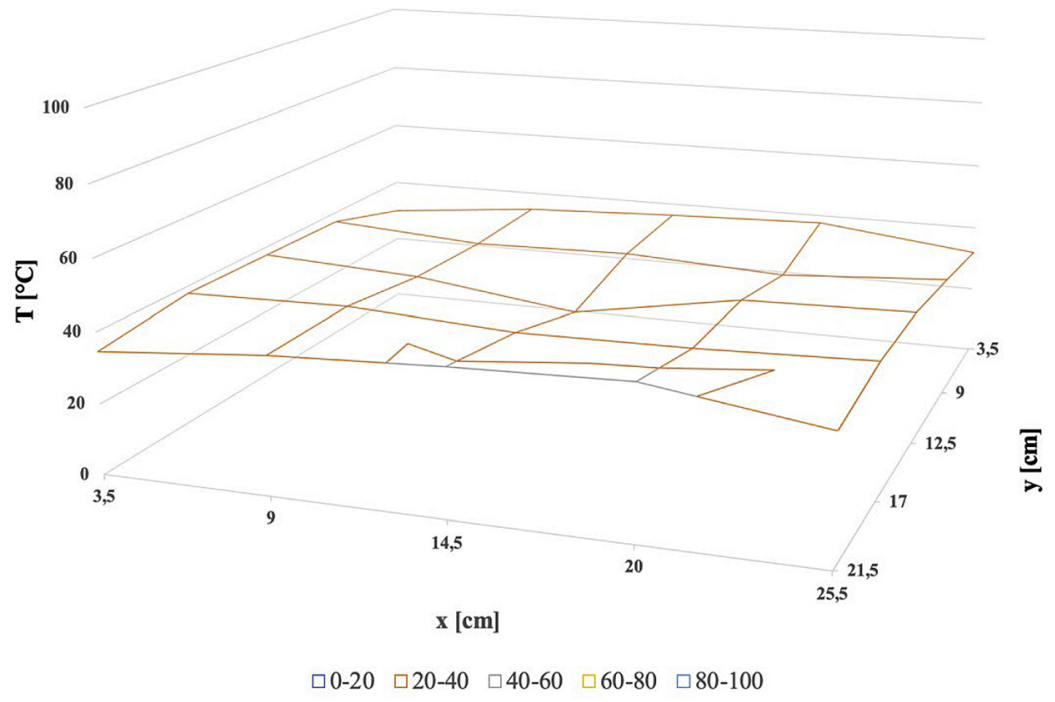

a)

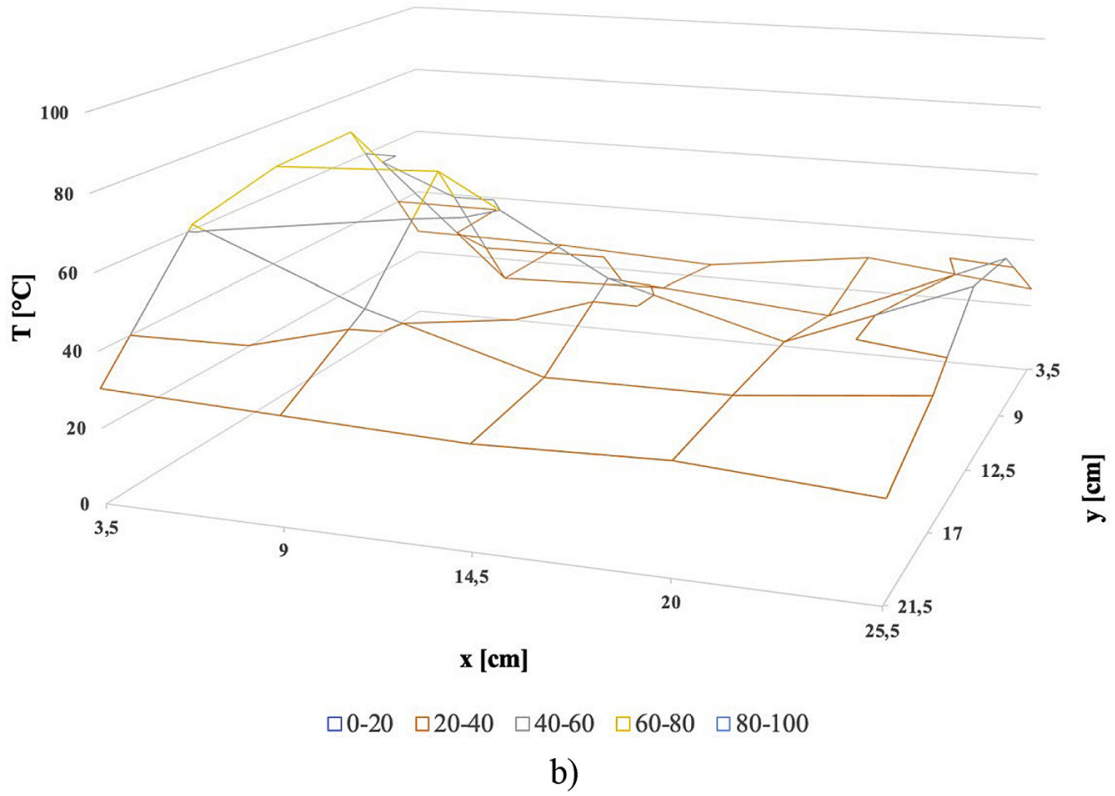

Fig. 4. Temperature distribution after 120 seconds of heating at mean output power of $350 \mathrm{~W}$ in cavity W (a) and cavity L (b) 


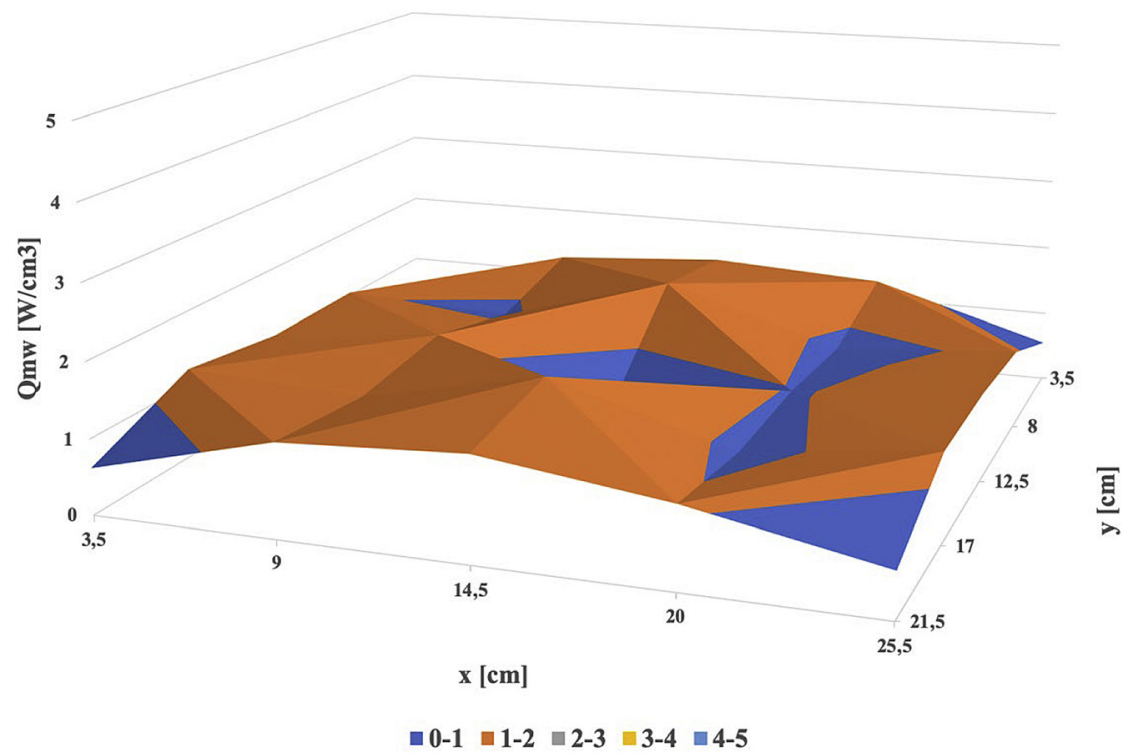

a)

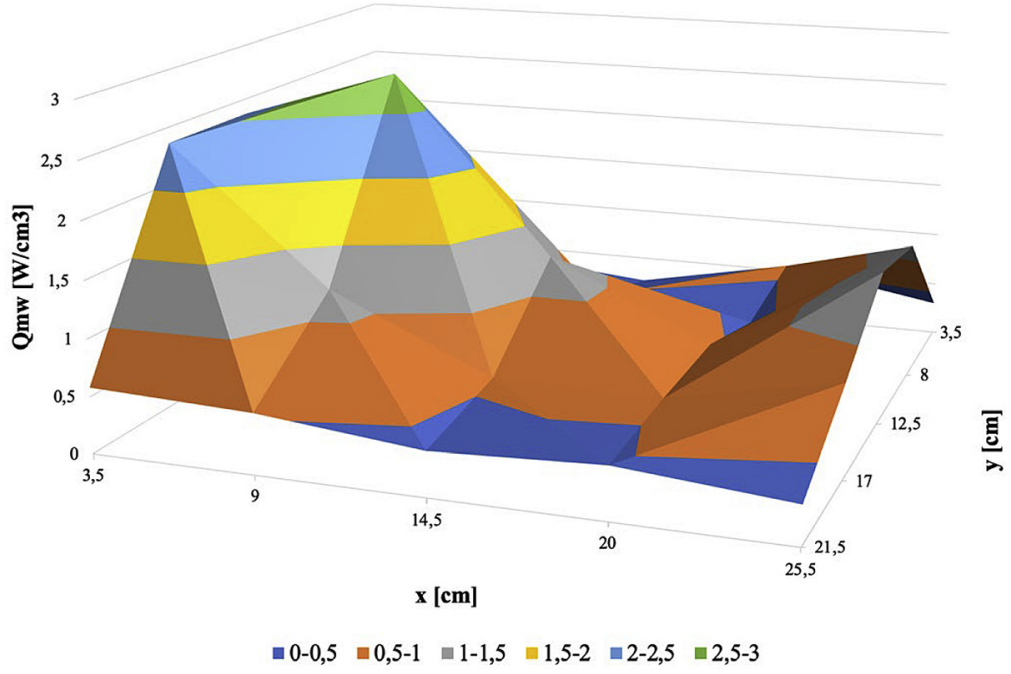

b)

Fig. 5. Specific microwave power distribution during exposure to an average microwave power of $350 \mathrm{~W}$ in cavity W (a) and cavity L (b) 
respectively. Every value of specific power in the figures is calculated at the time necessary for each of the beakers to reach a target temperature. The distribution is invariant with the chosen target temperature and it is time independent, in agreement with previous work (Pace et al., 2011).

\section{DISCUSSION}

The performances of the two investigated cavities changed in terms of temperature-time evolution and in temperature and specific power distribution.

Samples in cavity W were subjected to an intermittent heating, as proven by the alternate series of temperature ramps and plateaus along the time line (Fig. 3a), whereas the samples in cavity $\mathrm{L}$ exhibited a very linear trend of temperature versus time, position by position.
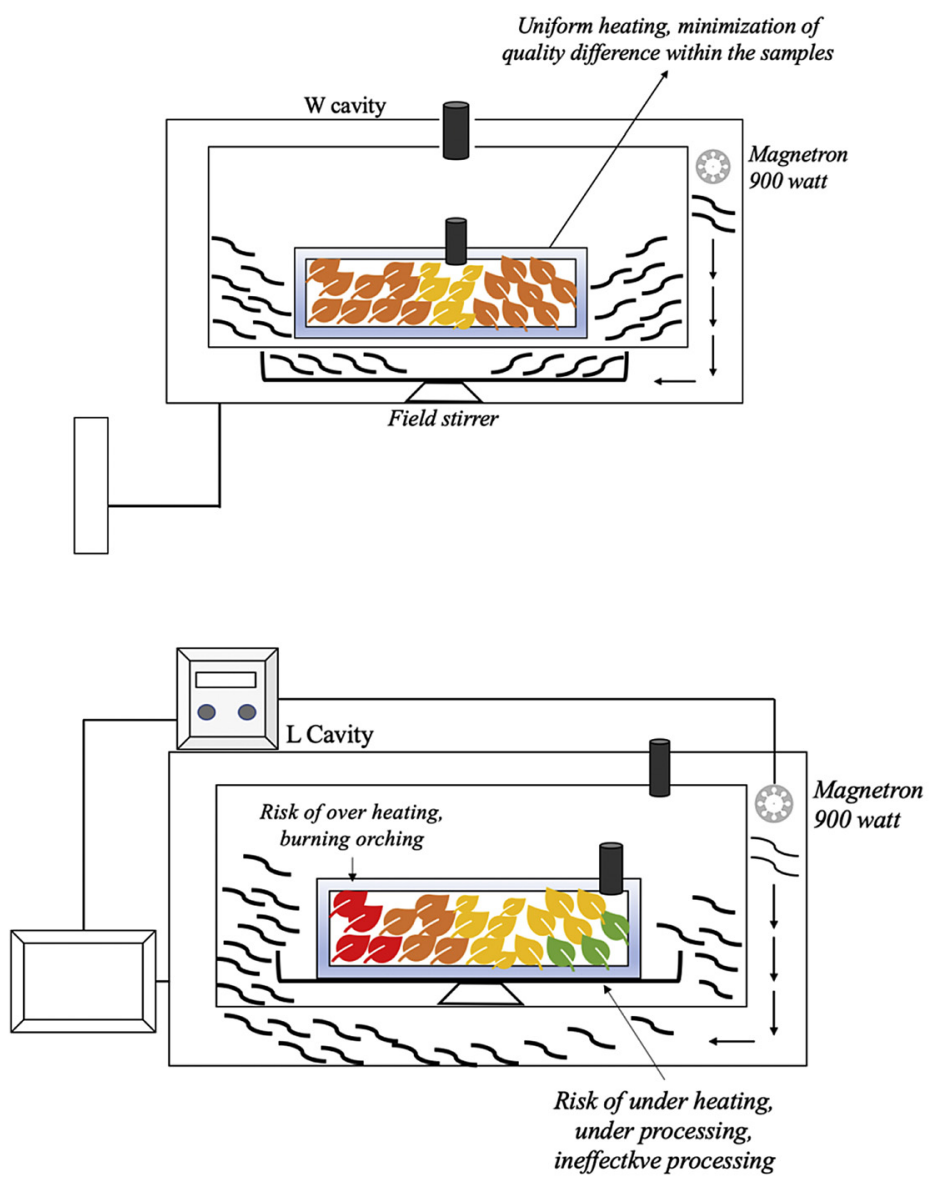

Fig. 6. Sketch of overall microwave power distribution in the two considered cavities, $\mathrm{W}$ and $\mathrm{L}$ 
The linearity of temperature vs time was also a proof that the hypothesis the heat balance equation was based on was correct, since both conductive and convective contribution to heat transfer in the water samples look negligible.

Temperature distribution in samples hold in cavity $\mathrm{W}$ resulted more even and uniform than in those hold in the case of cavity L, which instead resulted hot in some areas and much colder in others. Particularly, the beakers hold in cavity L, in the position further away from the waveguide port exhibited the higher temperature values.

The uneven distribution of sample temperatures in cavity $\mathrm{L}$ was reflected by the specific microwave power distribution, which appeared - for this cavity - non uniform and with points where the specific power ranges from $0.3 \cdot 10^{6}$ to $2.8 \cdot 10^{6} \mathrm{~W} / \mathrm{m}^{3}$, while in cavity $\mathrm{W}$ the specific microwave power distribution was uniform with values bounded between $1.0 \cdot 10^{6}$ and $2.0 \cdot 10^{6}$ $\mathrm{W} / \mathrm{m}^{3}$. Pace et al. (2011) found similar specific power distribution.

The linearly variable power supply thus allows a continuous, rather than a stop-and-go, heating of the loaded samples (Fig. 3), but the electromagnetic field stirrer appears to be effective in determining the evenness of heating. This may be of a crucial relevance when performing extraction from vegetable matrices, whose content of thermolabile, valuable compounds is very high.

It is clear that, in case of a specific power distribution like in cavity L, a sample hold in the area of higher microwave energy impact would suffer because of the over exposition to the waves, with consequent over heating or even arching or burning in some cases (De Bonis et al., 2015), like emphasized in Fig. 6. At same time, the samples treated in cavity L presented zones of the grid where there is risk of under heating (or under processing), with then scarce exploitation of the microwave energy and inefficient processing.

\section{CONCLUSIONS}

A simple but effective calorimetric method has been proposed to study the different temperature-time evolution and the spatial temperature and specific microwave power distribution in an array of samples hold in two microwave cavities, in agreement with the results obtained in previous work (Pace et al., 2011). These latter were different from each other by the microwaves distribution systems (cavity $\mathrm{W}$ was equipped with a field stirrer) and the microwave power supplier (cavity L was equipped with a continuous microwave power driver). Cavity W, even if operating with a classical on-off duty cycle, allowed to obtain uniform distribution of temperature and specific microwave power thanks to the presence of the stirrer of electromagnetic field just in front of the waveguide port. This feature is fundamental to achieve an efficient and homogeneous extraction from vegetables matrices, which are strongly affected by the density of microwaves inside the cavity. If the electromagnetic fields were not uniformly distributed, then it would mean that they will tend to absorb microwaves differently, resulting in different heat and consequently showing a different yield extraction (Datta and Anantheswaran, 2001).

Further work is needed to explore how the distribution of the specific microwave power changes with the planar position of the samples' array and, eventually, what benefit can be derived from the application of a continuous microwave power supplier to the cavity W, equipped with the electromagnetic field stirrer. 


\section{ACKNOWLEDGMENTS}

This work was done in the framework of Microexativa project, run at University of Salerno, Italy. "Progetto cofinanziato dall'Unione Europea, dallo Stato Italiano e dalla Regione Campania, nell'ambito del POR Campania FESR 2014-2020" Microexativa - Estrazione senza solvente, mediante microonde, di principi attivi per la cosmetica e la nutraceutica da matrici di Cannabis Sativa".

\section{REFERENCES}

Berk, Z. (2013). Food process engineering and technology. Chapt. 13. Elsevier, pp. 346-348, ISBN 978-0-12373660-4.

Chemat, F., Maryline, O., Vian, A., and Cravotto, G. (2012). Green extraction of natural products: concept and principles. International Journal of Molecular Sciences, 13(7): 8615-8627.

Curet, S., Rouaud, O., and Boillereaux, L. (2008). Microwave tempering and heating in a singlemode cavity: numerical and experimental investigations. Chemical Engineering and Processing, 47: 1656-1665.

Datta, A.K. and Anantheswaran, R.C. (2001). Handbook of microwave technology for food application. Marcel Dekker Inc., New York. 55, IV, 2.

De Bonis, M.V., Caccavale, P., and Ruocco, G. (2015). Convective control to microwave exposure of moist substrates. Part II: Model validation and application. International Journal of Heat and Mass Transfer, 86: 950-956.

Mendez-Sanchez, R.A., Kuhl, U., Barth, M., Lewenkopf, C.H., and Stockmann, H.J. (2003). Distribution of reflection coefficients in absorbing chaotic microwave cavities. Physical Review Letters, 91(17): $1741021-4$.

Pace, M., De Bonis, M.V., Marra, F., and Ruocco, G. (2011). Characterization of a combination oven prototype: Effects of microwave exposure and enhanced convection to local temperature rise in a moist substrate. Elsevier, International Communications in Heat and Mass Transfer, 38: 557-564.

Robinson, J., Kingman, S., Irvine, D., Licence, P., Smith, A., Dimitrakis, G., Obermayer, D., and Kappe, O. (2010). Understanding microwave heating effects in single mode type cavities-theory and experiment, Physical Chemistry Chemical Physics, 12: 4750-4758.

Open Access. This is an open-access article distributed under the terms of the Creative Commons Attribution-NonCommercial 4.0 International License (https://creativecommons.org/licenses/by-nc/4.0/), which permits unrestricted use, distribution, and reproduction in any medium for non-commercial purposes, provided the original author and source are credited, a link to the CC License is provided, and changes - if any - are indicated. 